\title{
Development of Echo-LPT for the study of particle-wall interactions in dense suspensions Milad Samie $^{1 *}$, Mohammad Reza Najjari ${ }^{1}$, Kai Zhang $^{1}$ and David E. Rival ${ }^{1}$
}

\author{
1: Department of Mechanical and Materials Engineering, Queen's University, Kingston, Canada \\ *corresponding author: m.samie@queensu.ca
}

\section{Introduction}

Examining the behaviour of dense suspensions has proven to be difficult, both experimentally and numerically. Using super water-absorbent polymer, PIV measurement was successfully conducted in a hydrogel suspension with a volume fraction (VF) of $\Phi=20 \%$ (see Zhang and Rival, 2018). However, due to the slightly refractive index mismatch, the image quality will degrade significantly as the particle loading of the hydrogel is increased. In order to achieve flow measurements in suspensions with high volume fractions, non-optical based techniques such as ultrasound imaging velocimetry (UIV) should be implemented. UIV has been developed for fluid dynamics applications and embraced by many researchers to study fluid flows (Gurung and Poelma, 2016; Jeronimo et al., 2019). Although, UIV provides useful information about the flow physics, it is unable to provide Lagrangian quantities such as particle trajectories, which is a key parameter to study entrainment and particle-wall interactions.

In this study, our goal is to investigate particle entrainment and particle-wall interactions in vortex rings created in dense suspensions using a Lagrangian method based on ultrasound imaging techniques. We have provided the validation of our UIV and echo Lagrangian Particle Tracking (eLPT) results. The tracking results will be further post-processed by a pathline extension method (Rosi and Rival, 2018). The resulting pathlines describe the lifespan of each fluid parcel within the measurement domain and can be used to extract path-dependent quantities, including particle approach velocity, incidence angle, particle-wall collision rate, particle residence time, and depletion efficiency.

\section{Experimental setup and methods}

We have performed several UIV and eLPT measurements using fundamental and harmonic imaging to measure vortex-ring flow fields. Figure 1(a) shows the experimental setup for our vortex-ring generator. A confined vortex ring was generated by a piston driving the flow in a cylinder with an inner diameter of $d_{0}=38.2 \mathrm{~mm}$. The piston is controlled by a linear traverse that moves at a constant speed of $U_{p}=0.16-0.32 \mathrm{~m} / \mathrm{s}$ over a stroke length of $L=191 \mathrm{~mm}\left(L / d_{0}=5\right)$. The generated vortex ring is confined within an acrylic tube with an inner diameter of $d=76.2 \mathrm{~mm}$ and a length of $457 \mathrm{~mm}$. To prepare the suspensions, various concentrations of SAP beads (LiquiBlock 2G-110 from Emerging Technologies, Inc.) were mixed with pure deionized water (DIW). These SAP beads have an absorption coefficient of $450 \mathrm{~g}_{\text {water }} / \mathrm{g}_{S A P}$ when mixed with DIW. Three working fluids were tested in this study: the pure DIW and suspensions with VFs of $\Phi=20 \%$ and $\Phi=40 \%$.

A Vantage 128 ultrasound system (Verasonics Inc.) with an L14-5/38 linear ultrasound probe (128 elements with $0.3 \mathrm{~mm}$ pixel pitch) was used to acquire plane-wave radio frequency (RF) signals. In the plane-wave imaging technique, all of the transducer's elements are activated together to form a planar acoustic wave. To increase the signal-to-noise ratio (SNR) of plane-wave images, a series of tilted plane-waves with different angles is used to reconstruct a single frame (Montaldo et al., 2009). In this study, we used a multi-planewaves (plane-wave compounding) approach with Verasonics' pixel-oriented reconstruction software to obtain high-quality B-mode images from RF data.

echo-Lagrangian Particle Tracking and Particle image velocimetry: We have acquired images for both eLPT and UIV. The particle density in eLPT was lower to allow for accurate distinction of individual particles. Acquired images were then processed using Davis 8.4 (LaVision) to obtain velocity maps. The 2D-PTV and PIV time-series correlation with a final window size of 32 pixels with 50\% overlap was used for eLPT and 
(a)
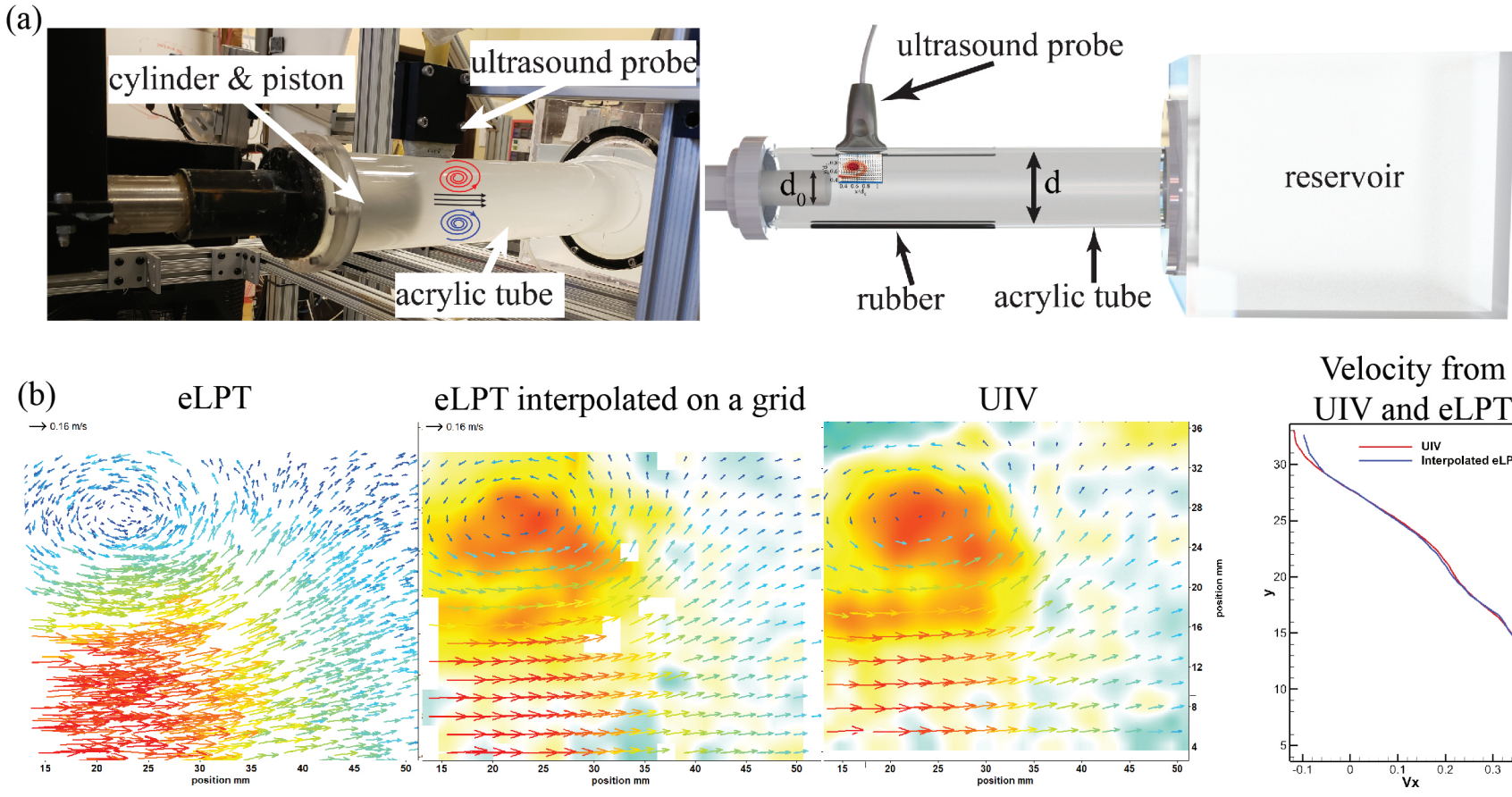

Velocity from UIV and eLPT

Figure 1: (a) Experimental setup of vortex-ring generator. (b) Comparison of instantaneous velocity vectors and vorticity maps for $\Phi=40 \%$ suspension obtained via eLPT and UIV.

UIV, respectively. The spatial resolution in the final UIV results were $1.6 \mathrm{~mm}$. The obtained UIV results were used as benchmark tests to validate the accuracy of eLPT measurements. Figure 1(b) shows the comparison of velocity and vorticity maps obtained with eLPT and UIV. Since the density of velocity vectors in eLPT was not as high as UIV, we merged 3 phase-locked instantaneous results from 3 different runs to obtain the eLPT vector field, which is then interpolated on a Cartesian grid. This figure demonstrates the accuracy of our eLPT results. Further work will be done to extend the short pathlines obtained from eLPT measurments, and to use the time history of each fluid parcel to elucidate the interactions between the suspended particles and the cylinder inner walls.

\section{References}

Arati Gurung and Christian Poelma. Measurement of turbulence statistics in single-phase and two-phase flows using ultrasound imaging velocimetry. Experiments in Fluids, 57(11):171, 2016. ISSN 0723-4864.

Mark D Jeronimo, Mohammad Reza Najjari, Kai Zhang, and David E Rival. Extraction of Particle Residence Time using echo-Lagrangian particle tracking. In 13th International Symposium on Particle Image Velocimetry, Munich, Germany. URL: https://athene-forschung. unibw. de/128884, 2019.

Gabriel Montaldo, Mickaël Tanter, Jérémy Bercoff, Nicolas Benech, and Mathias Fink. Coherent plane-wave compounding for very high frame rate ultrasonography and transient elastography. IEEE transactions on ultrasonics, ferroelectrics, and frequency control, 56(3):489-506, 2009. ISSN 0885-3010.

Giuseppe A Rosi and David E Rival. A Lagrangian perspective towards studying entrainment. Experiments in Fluids, 59(1):19, 2018. ISSN 0723-4864.

Kai Zhang and David E. Rival. Experimental study of turbulence decay in dense suspensions using indexmatched hydrogel particles. Physics of Fluids, 30(7), 2018. ISSN 10897666. doi: 10.1063/1.5031767. 